\title{
A CRITERION UTILITY CONVERSION TECHNIQUE FOR PROBABILISTIC LINGUISTIC MULTIPLE CRITERIA ANALYSIS IN EMERGENCY MANAGEMENT
}

\author{
Rui QIN®, Huchang LIAO ${ }^{\circledR}{ }^{*}$, Lisheng JIANG (1) \\ Business School, Sichuan University, Chengdu 610064, China
}

Received 02 November 2020; accepted 14 March 2021; first published online 05 July 2021

\begin{abstract}
In multiple criteria decision making (MCDM), the even swaps method uses the relationships of criteria to make trade-offs but the burdens of experts are heavy; the linear programming technique for multidimensional analysis of preference (LINMAP) method cannot deal with the inter-dependencies among criteria but the cognitive burdens of experts are low. Taking the advantages of both these methods, this study proposes a criterion utility conversion (CUC) technique to solve probabilistic linguistic MCDM problems given that the probabilistic linguistic term set (PLTS) can reflect the psychology of experts when making evaluations. The utility conversion process is first proposed based on the marginal utilities of criteria. Then, the criterion preference ratios of experts are refined from the utility conversion process. Based on the criterion preference ratios and the operations of PLTSs, the adjusted probabilistic linguistic expected values of alternatives are calculated. The consistency and inconsistency indexes of alternatives and criteria are defined to set up the linear programming used to work out the criterion preference ratios. An illustration about the selection of emergency logistics supplier is given to validate the proposed method. The comparative analysis indicates the low cognitive burden, high stability, and strong applicability of the proposed method.
\end{abstract}

Keywords: multiple criteria analysis, criterion utility conversion, probabilistic linguistic term set, emergency logistics supplier selection.

JEL Classification: C44, D70, D81, L83.

\section{Introduction}

As a multiple criteria decision-making (MCDM) model, the even swaps method (Hammond et al., 1993) ranks alternatives based on the trade-offs among criteria. In this method, experts need to give a fixed change on one criterion, and then, the compensatory changes on other criteria should be given to make the utilities of alternatives unchanged. Here, the utility conversion between the utility of the fixed change and compensatory change is done based

*Corresponding author. E-mail: liaohuchang@163.com

Copyright (c) 2021 The Author(s). Published by Vilnius Gediminas Technical University

This is an Open Access article distributed under the terms of the Creative Commons Attribution License (http://creativecommons. org/licenses/by/4.0/), which permits unrestricted use, distribution, and reproduction in any medium, provided the original author and source are credited. 
on the relations of criteria. For instance, there is a relation that the high quality has high cost. By such a relation, the trade-off between cost and quality is feasible. Using the trade-off, experts can make the values of a part of alternatives under certain criteria the same, leading to the convenience to compare alternatives. Due to this advantage, the even swaps method has attracted much attention, and its theoretical developments were mainly in three directions including the software development (Mustajoki \& Hämäläinen, 2005, 2007), the combination with other methods (Dereli \& Altun, 2012; Elahi \& Yu, 2012; Li \& Ma, 2008), and the consideration of biases (Lahtinen \& Hämäläinen, 2016; Lahtinen et al., 2020). Meanwhile, the even swaps method have been applied to many realms (Gomes et al., 2012; Kajanus et al., 2001; Milutinovic et al., 2018). The studies of the even swaps method on the software development and the combination with other methods extended the scope of applications of the even swaps method. The consideration of the biases of even swaps method improved the accuracy of even swaps method. Although the even swaps method has been improved, the abovementioned researches on the even swaps method required experts to participate in the whole decision-making process, resulting in the high burdens of experts.

The linear programming technique for multidimensional analysis of preference (LINMAP) (Srinivasan \& Shocker, 1973) is another useful MCDM method to rank alternatives. The core of this model is to use the evaluations and pairwise comparisons of alternatives to create the consistency and inconsistency indexes. With these indexes, linear programming can be set up to deduce the weights of criteria and the values of positive ideal solutions, so as to rank alternatives. A mass of achievements have been obtained in terms of the information environments (Li, 2008; Liao et al., 2019a; Lu et al., 2020), model structures (Chen, 2019; Haghighi et al., 2019), and the combinations with other methods (Kashef et al., 2018; Mehrabadi \& Boyaghchi, 2019). This method has also been applied to solve practical problems (Hamidzadeh et al., 2020; Yao, 2019; Zuo et al., 2019). The studies of the LINMAP method on different information environments and the combination with other methods extended the applications of the LINMAP method. The model structure improvement ameliorated the accuracy of LINMAP method. Although the LINMAP method has been improved, the aforementioned studies on the LINMAP method need the criteria to be independent, leading to the limitation of applying the LINMAP method.

As the decision-making environment becomes complicated, both crisp numbers and linguistic information are important. To express the complex decision-making environment, the probabilistic linguistic term set (PLTS) (Pang et al., 2016) consisting of multiple linguistic terms with probabilities was proposed. The PLTS allows experts to give information with both linguistic terms and probabilities, which facilitates the information expression of experts. Meanwhile, both quantitative and qualitative information could be included in the PLTS, thus enhancing the richness of information. Because of these advantages, the PLTS has attracted much attention and many MCDM methods within the context of PLTSs have been investigated (Lei et al., 2020; Liao et al., 2017, 2020; Mi et al., 2020; Pang et al., 2016; Zhang \& Xing, 2017). The operations of PLTSs are the base of the theoretical research related to PLTSs. A part of the studies on the operations of PLTSs (Liao et al., 2017; Pang et al., 2016; Zhang \& Xing, 2017) calculated PLTSs through subscript aggregation, resulting in the neglect of characteristics of human psychological risk preferences and the deviation 
of transformation of evaluation information (Wu \& Liao, 2019). To cope with these issues, Wu and Liao (2019) introduced the operations of PLTSs based on linguistic scale functions (Wang et al., 2014). Because the method of Wu and Liao was reasonable, it has been applied in other studies (Fang et al., 2021).

For the even swaps method, the burdens of experts are heavy as it needs experts to have trade-offs between all pairs of criteria and alternatives. When the alternatives and criteria are of large quantity, the cognitive load of experts will be high and thus might be easy to cause errors in results. In addition, the traditional LINMAP method did not consider the relations between criteria. When criteria have inter-dependencies, the simple weighted average might cause errors (Liao et al., 2019b). The studies of LINMAP method (Li, 2008; Liao et al., 2019a; Lu et al., 2020; Chen, 2019; Haghighiet al., 2019; Kashef et al., 2018; Mehrabadi \& Boyaghchi, 2019; Hamidzadeh et al., 2020; Yao, 2019; Zuo et al., 2019) just used the simple weights of criteria, so their results might be inaccurate. The even swaps method uses the relations of criteria to do the trade-offs, so the idea of trade-offs might be able to fit the challenge of the LINMAP method in terms of criterion relations. In the LINMAP method, since experts just need to give evaluation information and pairwise comparisons of alternatives for only one time to deduce the weights of criteria from linear programming, the challenge of experts' heavy burdens corresponding to the even swaps method might be released by this idea. Moreover, since the PLTS has advantages in expressing complex information, we use the PLTSs to depict the vague information in this study.

Motivated by these ideas, this study justifies the utility conversion process among criteria in the trade-off process. Then, a criterion preference ratio is refined from the utility conversion process. A linear programming problem is set up to work out the criterion preference ratios of experts. This proposed MCDM method is called the criterion utility conversion (CUC) technique.

The contributions of this study are summarized as follows:

(1) The criterion preference ratio is defined with probabilistic linguistic information. The utility conversion process is analyzed by the marginal utility of criteria. Then, the criterion preference ratio, which can be used to measure the preference of experts to different criteria, is defined. By the linguistic scale functions of PLTSs (Wu \& Liao, 2019), the adjusted probabilistic linguistic expected values of alternatives are given based on the criterion preference ratios, which can be used to rank alternatives.

(2) The PL-CUC method is proposed. The pairwise comparison sets of alternatives and criteria with truth degrees are given to define the consistency and inconsistency indexes of alternatives and criteria. A linear programming problem aiming to minimize the inconsistency indexes of alternatives and criteria is set up to work out the criterion preference ratios of experts. The PL-CUC method consisting of the criterion preference ratios, the linear programming, and the linguistic scale functions of PLTSs is then introduced.

(3) The PL-CUC method is applied to solve the emergency logistics supplier selection problem. The feasibility of the PL-CUC method is validated by a comparative analysis with four probabilistic linguistic MCDM methods. 
This study is organized as follows: In Section 1, we review the even swaps method, LINMAP method, and PLTS. Section 2 puts forward the PL-CUC method with probabilistic linguistic information. A case study about the selection of emergency logistics suppliers is introduced in Section 3. Concluding remarks are given in the last section.

\section{Preliminaries}

This section reviews the even swaps method and the LINMAP method. To facilitate the understanding of the probabilistic linguistic expected values, the PLTS and linguistic scale functions are also introduced.

\subsection{The even swaps method}

In decision making, alternative $a$ dominates alternative $b$ if and only if $b$ is not superior $a$ to under all criteria, and $a$ is better than $b$ under at least one criterion (Ben Abdelaziz et al., 1999). Because the dominance relations show the precedence relations of alternatives, we can use the dominance relations between alternatives to select the best alternative. However, in some complex cases of real world problems, $a$ might be worse than $b$ under some criteria when criteria are many, causing the situation where $a$ cannot totally dominate $b$, or saying, $a$ partially dominates $b$.

To solve the partially dominant problem, Hammond et al. (1993) proposed the even swaps method based on trade-offs. The trade-off relations between criteria are meant to make changes on the numerical values of criteria of one alternative so as to create another new alternative whose worth was equal to that of the original one. Here, the alternative worth could be understood as the alternative utility. Specifically, in the decision-making process, a decision-maker decides a fixed change of one alternative under one criterion. Such a fixed change on the criterion might alter the overall utility of the alternative. To keep the alternative utility constant, a change of the same alternative under another criterion could be made to compensate the alternative utility change caused by the fixed change on the previous criterion. Since the alternative utility change caused by the fixed change under the first criterion could be compensated by the compensatory change under the next criterion, the relations between these two criteria could be uncovered. A new alternative can be created based on these changes. Because the new alternative and the original one have the same utility, if the new alternative dominates another alternative, the original alternative also dominates this alternative. We use Example 1 to illustrate the trade-off process in the even swaps method.

Example 1. Mark needs to choose a part-time job. The criteria include the salary and commuting time. There are two jobs $a$ and $b$. The salary and commuting time of these two jobs are shown in Table 1.

Table 1 . Values of salary and commuting time of $a$ and $b$

\begin{tabular}{|l|c|c|}
\hline & $a$ & $b$ \\
\hline Salary (dollars per month) & 1000 & 1200 \\
\hline Commuting time (minutes) & 50 & 90 \\
\hline
\end{tabular}


We can find that the salary of $a$ is low but its commuting time is also low, so the dominance relation between $a$ and $b$ is unclear. In this case, Mark needs to make a trade-off between these two criteria. To use the even swaps method, the primary question is that how much salary he can cut for the commuting time of 40 minutes. Suppose that the answer is 400 dollars. Then, we can cut 40 minutes under the commuting time and 400 under the salary in $b$ to have a trade-off. After the trade-off, a new alternative $b^{\prime}$, which is equivalent to $b$, is obtained as shown in Table 2. Because the commuting time of $a$ and $b^{\prime}$ is the same and the salary of $a$ is higher than that of $b^{\prime}, a$ dominates $b^{\prime}$. Because $b$ and $b^{\prime}$ have the same utility for Mark, $a$ dominates $b$. Then, Mark can choose job $a$ because $a$ is better than $b$.

Table 2. The values after the trade-off

\begin{tabular}{|l|c|c|c|}
\hline & $a$ & $b^{\prime}$ & $b$ \\
\hline Salary (dollars per month) & 1000 & 800 & 1200 \\
\hline Commuting time (minutes) & 50 & 50 & 90 \\
\hline
\end{tabular}

After being introduced, the even swaps method achieved a series of theoretical developments which can be grouped into three categories. (1) The first group aimed to make the even swaps method easy to use. In this regard, Mustajoki and Hämäläinen (2005) constructed the preference programming based on the multi-attribute value theory to provide advices for the even-swaps process. Afterwards, Mustajoki and Hämäläinen (2007) proposed a web-based decision support tool to support the whole decision process. (2) The second group combined the even swaps method with other theories. For instance, Li and Ma (2008) constructed the even swaps process on spheres to consistently rank alternatives; in the absence of quantitative data but only with rough qualitative comparisons made by stakeholders, Elahi and Yu (2012) proposed a heuristic decision algorithm based on the even swaps method to analyze the possible results and obtain the final decision. Dereli and Altun (2012) developed a modified even swaps method to solve multi-issue negotiation problem. Based on the modified even swaps method, Altun and Dereli (2014) further proposed a way to eliminate unnecessary trade-offs by assessing the values of multi-issue offers, which further improved the operation efficiency. (3) The third group considered the biases of the even swaps method. Lahtinen and Hämäläinen (2016) proved that the path dependence existed in the even swaps method. Different decision paths were accompanied by corresponding errors affecting decision results. Lahtinen et al. (2020) put forward four new techniques to mitigate biases in the even swap process, improving the accuracy of results. Besides these theoretical developments, the even swaps method were also applied in several realms, such as the strategy selection in a rural enterprise (Kajanus et al., 2001), plastic surgery (Gomes et al., 2012), and site selection of reservoir (Milutinovic et al., 2018).

In general, decision-makers need to make trade-offs between all criteria for each pair of alternatives in the even swaps method. Such a complex process makes the even swaps method tedious and time-consuming. 


\subsection{The LINMAP method}

There are many MCDM methods (Adhami \& Ahmad, 2020; Haseli et al., 2020; Shaikh et al., 2020; Srinivasan \& Shocker, 1973), among which the LINMAP method proposed by Srinivasan and Shocker (1973) was well-known. The LINMAP method establishes a linear programming problem based on the decision matrices and pairwise comparisons of alternatives. The positive ideal solution and the weights of criteria were defined to get the distances between the positive ideal solution and all alternatives. Because the alternative close to the positive ideal solution have a good performance, the distances could be used to rank the alternatives. The consistency index and inconsistency index were defined to measure if the ranking was consistent with the pairwise comparisons of alternatives or not. It was noticeable that in the consistency index and inconsistency index, the positive ideal solution and the weights of criteria were unknown. The linear programming aiming to minimize the inconsistency index was built to work out the unknown parameters and then order the alternatives by the calculated distances. The significant advantage of the LINMAP method is that the cognitive load of experts is low. In the LINMAP method, the preference information is the pairwise comparisons of alternatives, and the experts do not need to give the pairwise comparisons of all alternatives. In addition, the weights of criteria are determined by the programming, which also release the work load of experts and the weights are objective.

Because of its advantages, the original LINMAP method has been extended to different environments by many scholars. For example, Li (2008) investigated the LINMAP method to solve decision-making problems in the intuitionistic fuzzy environment. Wang and Chen (2020) extended the LINMAP approach to the Pythagorean fuzzy environment to solve multiple criteria group decision making problems. Lu et al. (2020) proposed a novel three-phase LINMAP method in the dual hesitant fuzzy environment. The LINMAP method was also extended to the probabilistic linguistic environment by Liao et al. (2017). Moreover, scholars improved the model structure of the original LINMAP method. For instance, Haghighi et al. (2019) constructed a soft computing model based on the LINMAP approach. Chen (2019) established a parameter LINMAP model for multiple criteria group decision making. In addition, some studies combined the LINMAP method with other methods. For example, Kashef et al. (2018) erected an approach that combines the passive and active compensability multi-criteria analysis (PACMAN) with the LINMAP method to help decision-makers avoid identifying a complex parameter. Mehrabadi and Boyaghchi (2019) combined the LINMAP method and the technique for order of preference by similarity to ideal solution (TOPSIS) method to find the optimal performance of all generation system scenarios. The LINMAP method was also applied to solve practical problems, such as the property perception service quality assessment (Zuo et al., 2019), optimum design of spur gears (Yao, 2019) and recover flare gases (Hamidzadeh et al., 2020).

It is observed that in the original LINMAP method and its extensions, criteria are considered to be independent. However, it is common that criteria might influence each other. For example, the cost would be high when the quality is good. If there are inter-dependencies among criteria, the method with simple weighted average, which is the main operation of the LINMAP method, might cause errors (Liao et al., 2019b). 


\subsection{Probabilistic linguistic expected values}

In decision-making methods like the even swaps method and LINMAP method, for criteria like comfort and convenience, it is difficult for experts to give precise numbers. In this situation, linguistic terms like "good" and "bad" are useful. To compute the linguistic terms, Zadeh (1975) proposed the linguistic variables, which can use linguistic terms to represent the phenomena that are too complex or ill-defined to be described in precise numbers. In the case that people are hesitant among several linguistic terms, the hesitant fuzzy linguistic term set (Rodríguez et al., 2012) can be used to depict such hesitant and qualitative information. Besides, the linguistic terms might have different probabilities or importance. To represent such information, Pang et al. (2016) defined the PLTS where each linguistic term in the PLTS is associated with a probability. For example, people can give the information that the comfort degree is "high" or even "very high", and this information is a piece of hesitant fuzzy linguistic information. If the information is that the comfort degree is "high" with 0.7 probability or "very high" with 0.3 probability, it is a piece of probability linguistic information. The PLTS is flexible because of the linguistic term; in addition, the PLTS includes rich information in the form of linguistic terms and probabilities.

Definition 1. (Pang et al., 2016) Let $S=\left\{s_{\alpha} \mid \alpha=-\tau, \cdots 1,0,1 \cdots, \tau\right\}$ be an LTS. A PLTS on $S$ is

$$
h(p)=\left\{s^{k}\left(p^{k}\right) \mid s^{k} \in S, p^{k} \geq 0, k=1,2, \cdots K, \sum_{k=1}^{K} p^{k} \leq 1\right\},
$$

where $K$ is the number of linguistic terms in $h(p) . s^{k}\left(p^{k}\right)$ is the $k$ th linguistic term $s^{k}$ with the corresponding probability $p^{k}$.

There are many researches on the extensions and applications of PLTSs. Liao et al. (2017, 2019a) introduced the probabilistic linguistic LINMAP method and the probabilistic linguistic ELECTRE III method for MCDM. Wu et al. (2018) proposed the probabilistic linguistic multiplicative multi-objective optimization by ratio analysis (PL-MULTIMOORA) method with the combined weights and applied it to an investment problem. Wen et al. (2019) extended the combined compromise solution (CoCoSo) method to probabilistic linguistic environment to solve a cold chain supplier selection problem. Wei et al. (2020) proposed a probabilistic linguistic group decision making method based on the grey correlation theory to solve a location problem of electric vehicle charging stations. The probabilistic linguistic decision-making methods have been applied to many areas, such as green supplier selection (Lei et al., 2020), edge computing (Lin et al., 2019) and cloud-based enterprise resource planning selection (Chen et al., 2019).

The operations of PLTSs are the foundation of probabilistic linguistic MCDM methods. Some researches directly used the subscripts of linguistic terms to operate PLTSs (Liao et al., 2017; Pang et al., 2016; Zhang \& Xing, 2017). The operations based on subscripts did not consider the characteristics of risk preference psychology of experts and ignored the existence of unbalanced linguistic terms, leading to the defects of calculation results (Wu \& Liao, 2019). To solve these defects, Wu and Liao (2019) calculated PLTSs by linguistic scale functions. The linguistic scale functions transform linguistic terms to their corresponding semantics considering people's psychology. The linguistic scale functions (Wang et al., 2014). have three types of monotonically increasing functions: 
1) When people have no risk preference and the semantics of linguistic term sets are uniformly distributed, the linguistic scale function can be represented as:

$$
f\left(s_{\alpha}\right)=(\alpha+\tau) / 2 \tau \text {. }
$$

2) When people have no risk preference and the semantics of linguistic term sets are unevenly distributed, the linguistic scale function can be denoted as:

$$
f\left(s_{\alpha}\right)=\frac{\tau^{\gamma}-(-\alpha)^{\gamma}}{2 \tau^{\gamma}} \times 1_{\{\alpha \in[-\tau, 0]\}}+\frac{\tau^{\psi}+(\alpha)^{\psi}}{2 \tau^{\psi}} \times 1_{\{\alpha \in(0, \tau]\}},
$$

where $1_{\{\alpha \in[-\tau, 0]\}}$ means than if $\alpha \in[-\tau, 0], 1_{\{\alpha \in[-\tau, 0]\}}=1$. If not, $1_{\{\alpha \in[-\tau, 0]\}}=0 . \gamma$ and $\psi$ are two parameters determined according to specific problems with $\gamma, \psi \in(0,1]$.

3) When people have risk preference and the semantics of linguistic term sets are unevenly distributed, the linguistic scale function can be given as:

$$
f\left(s_{\alpha}\right)=\frac{t^{\tau}-t^{-\alpha}}{2 t^{\tau}-2} \times 1_{\{\alpha \in[-\tau, 0]\}}+\frac{\mu^{\tau}+\mu^{\alpha}-2}{2 \mu^{\tau}-2} \times 1_{\{\alpha \in(0, \tau]\}},
$$

where $t>1$ refers to the parameter of risk preference for the linguistic terms with bad meanings, and $\mu>1$ is the parameter of risk preference for the linguistic terms with good meanings.

With different linguistic scale functions and probabilities, the probabilistic linguistic expected value of PLTS $h(p)$ was proposed as (Pang et al., 2016):

$$
E(h(p))=\sum_{k=1}^{K}\left(f\left(s^{k}\right) \cdot p^{k}\right)
$$

The probabilistic linguistic expected value $E(h(p))$ considers the situation of different semantics and probability values, such that the information is not lost in the calculation of PLTSs (Wu \& Liao, 2019). The probabilistic linguistic expected value has been applied in other studies, such as the evidential reasoning approach (Fang et al., 2021). In this paper, we also use the probabilistic linguistic expected values to calculate PLTSs.

\section{The CUC method with probabilistic linguistic information}

As discussed in Section 1, the LINMAP method cannot deal with the inter-dependencies among criteria but the cognitive load of experts is low. For the even swaps method, the trade-off process uses the inter-dependencies among criteria to analyze but it is tedious and time-consuming. Because the LINMAP method and even swaps method might overcome each other's drawback, the criteria utility conversion process and criterion preference ratios motivated by the even swaps method are proposed to deal with the inter-dependencies among criteria, and a linear programming problem inspired by the LINMAP method is set up to get the criterion preference ratios. The utility conversion process, criterion preference ratio, and the linear programming consist of the CUC method. Because the PLTS is a useful tool to express complex human evaluations having the cognitive ambiguity, we use PLTSs to express people's evaluations in the proposed method. Because the probabilistic linguistic expected values are accurate in calculation, this study uses the probabilistic linguistic scale functions to calculate PLTSs. 


\subsection{The criterion preference ratio}

A probabilistic linguistic MCDM problem consists of a finite set of alternatives, $a_{i}(i=1,2, \cdots, m)$, and a set of criteria, $c_{j}(j=1,2, \cdots, n)$. An expert gives the evaluation in a PLTS $h_{i j}(p)=$ $\left\{s_{i j}^{k}\left(p_{i j}^{k}\right) \mid s_{i j}^{k} \in S, p_{i j}^{k} \geq 0, k=1,2, \cdots K, \sum_{k=1}^{K} p_{i j}^{k} \leq 1\right\}$ to show the performance of alternative $a_{i}$ under criterion $c_{j}$. Then, the probabilistic linguistic evaluation matrix is constructed as $H=\left[h_{i j}(p)\right]_{m \times n}$. The probabilistic linguistic expected value matrix is $\mathbf{E}=\left[E\left(h_{i j}(p)\right)\right]_{m \times n}$. To make calculations easy, we suppose that the utility functions of criteria are linear functions.

Assumption 1. The marginal utility of criterion is a constant.

Figure 1 shows the linear utility functions of $c_{j_{1}}$ and $c_{j_{2}}$. In Figure 2, for a variation of utility $\Delta_{U}$, the change of criterion $c_{j_{1}}$ might be $\Delta_{j_{1}}$, and that of $c_{j_{2}}$ might be $\Delta_{j_{2}}$. The marginal utilities of criteria $c_{j_{1}}$ and $c_{j_{2}}$ are $\chi_{j_{1}}=\Delta_{U} / \Delta_{j_{1}}$ and $\chi_{j_{2}}=\Delta_{U} / \Delta_{j_{2}}$, respectively.

In the even swaps method, the utility variations of criteria influence the alternative utility. Since people are rational, the sum of utility variations of criteria is equal to that of the alternative. Then, we have the following assumption.

Assumption 2. (Lahtinen et al., 2020) Suppose that for alternative $a_{i}$, the utility variation of criterion $c_{j}$ is $\Delta_{U j}^{i}$. The utility variation of alternative is $\Delta_{U}^{i}=\sum_{j} \Delta_{U j}^{i}$.

For a fixed change $\Delta_{j_{1}}$ of $c_{j_{1}}$, it is known from Assumption 1 that the utility variation of $c_{j_{1}}$ is $\Delta_{U j_{1}}=\chi_{j_{1}} \times \Delta_{j_{1}}$. Because of Assumption 2, the utility variation of the alternative is also $\Delta_{U j_{1}}$. To make the alternative utility constant, we need to have a compensatory variation of the utility on another criterion $c_{j_{2}}$. The utility variation of $c_{j_{1}}$ is equal to that of $c_{j_{2}}$ if $\chi_{j_{1}} \times \Delta_{j_{1}}=\chi_{j_{2}} \times \Delta_{j_{2}}$. Then, the compensatory change of $c_{j_{2}}$ is $\Delta_{j_{2}}=\left(\chi_{j_{1}} / \chi_{j_{2}}\right) \times \Delta_{j_{1}}$. This is a utility conversion process from $\Delta_{j_{1}}$ of $c_{j_{1}}$ to $\Delta_{j_{2}}$ of $c_{j_{2}}$, which is shown in Figure 2 .

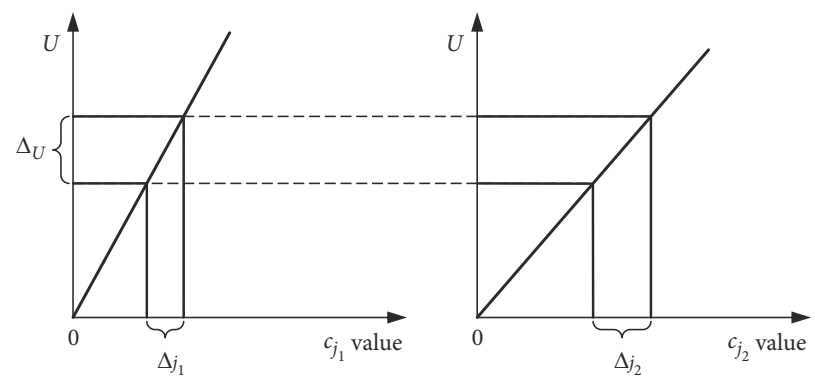

Figure 1 . The linear utility functions of $c_{j_{1}}$ and $c_{j_{2}}$

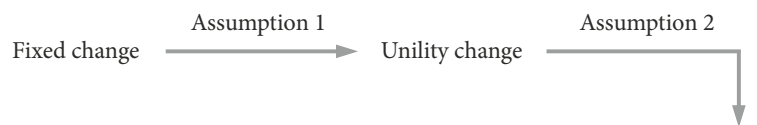

Alternative utility

Figure 2 . The utility conversion process 
Theorem 1. When $\chi_{j_{1}} / \chi_{j_{2}}<1$, people prefer $c_{j_{2}}$ to $c_{j_{1}}$. When $\chi_{j_{1}} / \chi_{j_{2}}=1, c_{j_{2}}$ and $c_{j_{1}}$ are the same for people. When $\chi_{j_{1}} / \chi_{j_{2}}>1$, people prefer $c_{j_{1}}$ to $c_{j_{2}}$.

Proof. $\chi_{j_{1}} / \chi_{j_{2}}$ shows the criterion preference of an expert. When $\chi_{j_{1}} / \chi_{j_{2}}$ is smaller than 1 , it means that one unit change of $c_{j_{1}}$ only causes less than one unit compensatory change of $c_{j_{2}}$. This unequal change shows that the utility of one unit change of $c_{j_{1}}$ is less than that of $c_{j_{2}}$, so it can be inferred that the expert prefers $c_{j_{2}}$ to $c_{j_{1}}$. For the similar method, we can proof the situations where $\chi_{j_{1}} / \chi_{j_{2}}=1$ or $\chi_{j_{1}} / \chi_{j_{2}}>1$.

By the utility conversion of criteria, the ratio $\chi_{j_{1}} / \chi_{j_{2}}$ can be used to express the preference relations of criteria.

Definition 2. The criterion preference ratio of $c_{j_{1}}$ and $c_{j_{2}}$ is

$$
\kappa_{j_{1}, j_{2}}=\chi_{j_{1}} / \chi_{j_{2}}
$$

where $\chi_{j_{1}}$ and $\chi_{j_{2}}$ are the marginal utilities of criteria $c_{j_{1}}$ and $c_{j_{2}}$, respectively. $\kappa_{j_{1}, j_{2}}>0$.

Theorem 2. For any three criteria $c_{1}, c_{2}$ and $c_{3}$, we have $\kappa_{j_{1}, j_{3}}=\kappa_{j_{1}, j_{2}} \times \kappa_{j_{2}, j_{3}}$.

Proof. $\kappa_{j_{1}, j_{2}} \times \kappa_{j_{2}, j_{3}}=\left(\chi_{j_{1}} / \chi_{j_{2}}\right) \times\left(\chi_{j_{2}} / \chi_{j_{3}}\right)=\chi_{j_{1}} / \chi_{j_{3}}=\kappa_{j_{1}, j_{3}}$. This completes the proof.

In the probabilistic linguistic expected value matrix $\mathbf{E}=\left[E\left(h_{i j}(p)\right)\right]_{m \times n}$, if we convert $E\left(h_{i j}(p)\right)(j \neq 1)$ to the first criterion $c_{1}$ by the utility conversion process, the change on $c_{1}$ is $\Delta_{E_{i j}}=E\left(h_{i j}(p)\right) \times \kappa_{j, 1} \cdot \kappa_{j, 1}$ is the conversion ratio of utility from $c_{j}$ to $c_{1}$. When the fixed change of $c_{j}$ is one unit, the compensation change on $c_{1}$ is $\kappa_{j, 1}$. In the same way, all criteria $c_{j}(j \neq 1)$ can be converted to $c_{1}$. The adjusted probabilistic linguistic expected value of $a_{i}$ under $c_{1}$ is

$$
\widehat{E}\left(h_{i 1}(p)\right)=E\left(h_{i 1}(p)\right)+\sum_{j>1}^{n} \rho_{j, 1} \times E\left(h_{i j}(p)\right) \times \kappa_{j, 1} \text {, }
$$

where $\rho_{j, 1}$ is the criterion type parameter. If $c_{1}$ and $c_{j}$ are cost criteria, the reduction on $c_{j}$ might cause an increase of the utility of the alternative. To keep the alternative utility constant, $c_{1}$ should make an increase to create a reduction of the utility of the alternative. Hence, when $c_{1}$ and $c_{j}$ are cost criteria, $\rho_{j, 1}=1$. With the similar inference, when $c_{1}$ and $c_{j}$ are benefit criteria, $\rho_{j, 1}=1$. To sum up, if $c_{1}$ and $c_{j}$ are the same criterion type, $\rho_{j, 1}=1$. If not, $\rho_{j, 1}=-1$. It is noted that, in Eq. (7), we can choose not only $c_{1}$ but also any criterion in $c_{j}(j=1,2, \cdots, n)$. The criterion preference ratios and weights are two different concepts. The meanings of them are completely different. The uses of these two methods are not the same. For the weights, the weighted sum is a common way for aggregation, but the interdependencies among criteria might cause errors (Liao et al., 2019b). For the criteria preference ratios, by the inter-dependencies among criteria, the aggregation of evaluations can be done by the utility conversion process.

By Eq. (7), each alternative $a_{i}$ has only one probabilistic linguistic expected value $\widehat{E}\left(h_{i 1}(p)\right)$. Then, we can rank the alternatives $a_{i}(i=1,2, \cdots, m)$ in the order of their adjusted probabilistic linguistic expected values $\widehat{E}\left(h_{i 1}(p)\right)(i=1,2, \cdots, m)$. If $c_{1}$ is a cost criterion, it is in descending order of $\widehat{E}\left(h_{i 1}(p)\right)(i=1,2, \cdots, m)$. If $c_{1}$ is a benefit criterion, it is in ascending order. 


\subsection{The linear programming to get the criterion preference ratios}

For an expert, it is difficult to accurately give $\kappa_{j, 1}$. Motivated by the LINMAP method, this study works out the criterion preference ratios $\kappa_{j, 1}(j=\{2,3, \cdots, n\})$ by the linear programming with the information of alternatives and criteria.

To set up the programming, the expert is required to give the pairwise comparisons of alternatives and criteria with truth degrees (Li \& Wan, 2014) as follows: 1) The pairwise comparison set of criteria: $\left.\aleph^{c}=\left\{<\left(c_{j_{1}}, c_{j_{2}}\right), R_{j_{1}, j_{2}}^{c}\right\rangle\left(j_{1} \neq j_{2} ; j_{1}, j_{2}=1,2, \cdots, n\right)\right\}$, which implies that the expert prefers $c_{j_{1}}$ to $c_{j_{2}}$ with the truth degree $R_{j_{1}, j_{2}}^{c}$; 2) The pairwise comparison set of alternatives: $\aleph^{a}=\left\{\left\langle\left(a_{i_{1}}, a_{i_{2}}\right), R_{i_{1}, i_{2}}^{a}\right\rangle\left(i_{1} \neq i_{2} ; i_{1}, i_{2}=1,2, \cdots, m\right)\right\}$, which implies that the expert prefers $a_{i_{1}}$ to $a_{i_{2}}$ with the truth degree $R_{i_{1}, i_{2}}^{a} \cdot R_{j_{1}, j_{2}}^{c}$ and $R_{i_{1}, i_{2}}^{a}$ belong to $(0,1]$. The higher truth degree means the higher reliability of the pairwise comparison.

When the expert gives $<\left(c_{j_{1}}, c_{j_{2}}\right), R_{j_{1}, j_{2}}^{c}>$, by Theorem 1 , it is known that $\kappa_{j_{1}, j_{2}}>1$. If the results of the linear programming model are consistent with $<\left(c_{j_{1}}, c_{j_{2}}\right), R_{j_{1}, j_{2}}^{c}>$, the criterion consistency index can be expressed as $\Phi_{j_{1}, j_{2}}^{c+}=\left(\kappa_{j_{1}, j_{2}}-1\right) \times R_{c}$. If it is not consistent, $\Phi_{j_{1}, j_{2}}^{c+}$ is 0 . A high $\Phi_{j_{1}, j_{2}}^{c+}$ indicates that the results of the linear programming model have a high consistent degree with the pairwise comparisons of criteria. In a nutshell, the criterion consistency index of $\left\langle\left(c_{j_{1}}, c_{j_{2}}\right), R_{j_{1}, j_{2}}^{c}>\right.$ can be expressed as:

$$
\Phi_{j_{1}, j_{2}}^{c+}=\max \left\{\left(\kappa_{j_{1}, j_{2}}-1\right) \times R_{j_{1}, j_{2}}^{c}, 0\right\} .
$$

Similarly, the criterion inconsistency index of $\left\langle\left(c_{j_{1}}, c_{j_{2}}\right), R_{j_{1}, j_{2}}^{c}>\right.$ is shown as:

$$
\Phi_{j_{1}, j_{2}}^{c-}=\max \left\{\left(1-\kappa_{j_{1}, j_{2}}\right) \times R_{j_{1}, j_{2}}^{c}, 0\right\} \text {. }
$$

For $\Phi^{c}$, the total criterion consistency index is

$$
\Phi^{c+}=\sum_{\forall\left(c_{j_{1}}, c_{j_{2}}\right) \in \aleph^{c}} \max \left\{\left(\kappa_{j_{1}, j_{2}}-1\right) \times R_{j_{1}, j_{2}}^{c}, 0\right\} .
$$

The total criterion inconsistency index is

$$
\Phi^{c-}=\sum_{\forall\left(c_{j_{1}}, c_{j_{2}}\right) \in \aleph^{c}} \max \left\{\left(1-\kappa_{j_{1}, j_{2}}\right) \times R_{j_{1}, j_{2}}^{c}, 0\right\} .
$$

Theorem 3. For a pairwise comparison $\left\langle\left(c_{j_{1}}, c_{j_{2}}\right), R_{j_{1}, j_{2}}^{c}>\right.$, one of $\Phi_{j_{1}, j_{2}}^{c+}$ and $\Phi_{j_{1}, j_{2}}^{c-}$ is 0 .

Proof. It is clear that $\Phi_{j_{1}, j_{2}}^{c+}, \Phi_{j_{1}, j_{2}}^{c-} \geq 0$. If $\Phi_{j_{1}, j_{2}}^{c+}>0, \kappa_{j_{1}, j_{2}}>1$, so $\Phi_{j_{1}, j_{2}}^{c-}=\max \left\{\left(1-\kappa_{j_{1}, j_{2}}\right) \times\right.$ $\left.R_{j_{1}, j_{2}}^{c}, 0\right\}=0$. If $\Phi_{j_{1}, j_{2}}^{c+}=0, \kappa_{j_{1}, j_{2}} \leq 1$, so $\Phi_{j_{1}, j_{2}}^{c-}=\max \left\{\left(1-\kappa_{j_{1}, j_{2}}\right) \times R_{j_{1}, j_{2}}^{c}, 0\right\}>0$. This completes the proof.

When the expert gives $<\left(a_{i_{1}}, a_{i_{2}}\right), R_{i_{1}, i_{2}}^{a}>$, by Eq. (7), it is known that $\varsigma \times\left(\widehat{E}\left(h_{i_{1}, 1}(p)\right)-\widehat{E}\left(h_{i_{2}, 1}(p)\right)\right)>0$ where $\varsigma=1$ if $c_{1}$ is a benefit criterion, and $\varsigma=-1$ if $c_{1}$ is a cost criterion. If the results of the linear programming are consistent with $<\left(a_{i_{1}}, a_{i_{2}}\right), R_{i_{1}, i_{2}}^{a}>$, the alternative consistency index is $\Phi_{i_{1}, i_{2}}^{a+}=\left(\varsigma \times\left(\widehat{E}\left(h_{i_{1}, 1}(p)\right)-\widehat{E}\left(h_{i_{2}, 1}(p)\right)\right)\right) \times R_{i_{1}, i_{2}}^{a}$. If it is not consistent, $\Phi_{i_{1}, i_{2}}^{a+}$ is 0 . In conclusion, the alternative consistency index of $<\left(a_{i_{1}}, a_{i_{2}}\right), R_{i_{1}, i_{2}}^{a}>$ can be expressed as:

$$
\Phi_{i_{1}, i_{2}}^{a+}=\max \left\{\left(\varsigma \times\left(\widehat{E}\left(h_{i_{1}, 1}(p)\right)-\widehat{E}\left(h_{i_{2}, 1}(p)\right)\right)\right) \times R_{i_{1}, i_{2}}^{a}, 0\right\} .
$$


Similarly, the alternative inconsistency index of $\left\langle\left(a_{i_{1}}, a_{i_{2}}\right), R_{i_{1}, i_{2}}^{a}>\right.$ is shown as:

$$
\Phi_{i_{1}, i_{2}}^{a-}=\max \left\{\left(\varsigma \times\left(\widehat{E}\left(h_{i_{2}, 1}(p)\right)-\widehat{E}\left(h_{i_{1}, 1}(p)\right)\right)\right) \times R_{i_{1}, i_{2}}^{a}, 0\right\} .
$$

For $\Phi^{a}$, the total alternative consistency index is

$$
\Phi^{a+}=\sum_{\forall\left(a_{i_{1}}, a_{i_{2}}\right) \in \aleph^{a}} \max \left\{\left(\varsigma \times\left(\widehat{E}\left(h_{i_{1}, 1}(p)\right)-\widehat{E}\left(h_{i_{2}, 1}(p)\right)\right)\right) \times R_{i_{1}, i_{2}}^{a}, 0\right\} .
$$

The total alternative inconsistency index is

$$
\Phi^{a-}=\sum_{\forall\left(a_{i_{1}}, a_{i_{2}}\right) \in \aleph^{a}} \max \left\{\left(\varsigma \times\left(\widehat{E}\left(h_{i_{2}, 1}(p)\right)-\widehat{E}\left(h_{i_{1}, 1}(p)\right)\right)\right) \times R_{i_{1}, i_{2}}^{a}, 0\right\} .
$$

Theorem 4. For a pairwise comparison $\left\langle\left(a_{i_{1}}, a_{i_{2}}\right), R_{i_{1}, i_{2}}^{a}>\right.$, one of $\Phi_{i_{1}, i_{2}}^{a+}$ and $\Phi_{i_{1}, i_{2}}^{a-}$ is 0 .

The proof of Theorem 4 is similar to that of Theorem 3 .

Afterwards, the linear programming can be established to minimize the sum of the total alternative inconsistency index and total criterion inconsistency index.

Model 1

$$
\begin{gathered}
\min \Phi^{a-}+\Phi^{c-} \\
\text { s.t.: }\left\{\begin{array}{l}
\Phi^{a+}-\Phi^{a-}>\varepsilon^{a} \\
\Phi^{c+}-\Phi^{c-}>\varepsilon^{c} \\
\kappa_{j, 1}>0, \forall j \in\{2, \cdots, n\}
\end{array} .\right.
\end{gathered}
$$

$\Phi^{a+}-\Phi^{a-}>\varepsilon^{a}$ and $\Phi^{c+}-\Phi^{c-}>\varepsilon^{c}$ mean that the consistency indexes should be larger than the inconsistency indexes associated with the thresholds $\varepsilon^{a}$ and $\varepsilon^{c}$ given by the expert.

By Model 1, $\kappa_{j, 1}(j \in\{2, \cdots, n\})$ and $\widehat{E}\left(h_{i 1}(p)\right)(i=1,2, \cdots, m)$ can be worked out. According to $\widehat{E}\left(h_{i 1}(p)\right)$, the best alternative can be selected, and the probabilistic linguistic MCDM problem is solved.

To facilitate the application, we summarize the algorithm of the CUC method below.

\section{Algorithm 1. (The CUC method with PLTSs)}

Step 1. The expert $e$ gives the evaluation information of alternatives $a_{i}(i=1,2, \cdots, m)$ under criteria $c_{j}(j=1,2, \cdots, n)$ in PLTSs. Then, we set up a probabilistic linguistic evaluation matrix $H=\left[h_{i j}(p)\right]_{m \times n}$. Let expert give the pairwise comparison sets $\Phi^{a}$ and $\Phi^{c}$ on alternatives and criteria, respectively. The thresholds $\varepsilon^{a}$ and $\varepsilon^{c}$ should also be determined. Go to the next step.

Step 2. The probabilistic linguistic expected value matrix $\mathbf{E}=\left[E\left(h_{i j}(p)\right)\right]_{m \times n}$ of $H=\left[h_{i j}(p)\right]_{m \times n}$ is established by Eqs. (2)-(5). Go to the next step.

Step 3. Define the criterion preference ratios $\kappa_{j, 1}(j \in\{2, \cdots, n\})$ of $c_{j}$ and $c_{1}$. Calculate the adjusted probabilistic linguistic expected values $E\left(h_{i 1}(p)\right)(i=1,2, \cdots, m)$ by Eq. (7). Go to the next step.

Step 4. Calculate the total criterion consistency index $\Phi^{c+}$ and the total criterion inconsistency index $\Phi^{c-}$ by Eqs. (10) and (11). Calculate the total alternative consistency index $\Phi^{a+}$ and the total alternative inconsistency index $\Phi^{a-}$ by Eqs (14) and (15). Then, set up Model 1. Go to the next step. 
Step 5. Solve Model 1 to get $\kappa_{j, 1}(j \in\{2, \cdots, n\})$. Work out the values of $\widehat{E}\left(h_{i 1}(p)\right)(i=1,2, \cdots, m)$ to rank the alternatives. End.

In Algorithm 1, the expert firstly evaluates the alternatives in PLTSs, and then sets up the probabilistic linguistic evaluation matrix. The expert also needs to give the pairwise comparison sets of criteria and alternatives, and the thresholds for Model 1. Secondly, the probabilistic linguistic expected value matrix can be set up based on the probabilistic linguistic evaluation matrix. Thirdly, the criterion preference ratios of the first criterion and other criteria should be defined. Based on the ratios, the adjusted probabilistic linguistic expected values corresponding to the first criterion of all alternatives can be calculated. Fourthly, the consistency indexes and inconsistency indexes of criteria and alternatives can be calculated to set up Model 1. Finally, the criterion preference ratios are got by solving Model 1 . The alternatives can be ranked by their adjusted probabilistic linguistic expected values worked out by the criterion preference ratios.

\section{Case study: logistics supplier selection for emergency management}

\subsection{Case description}

In recent decades, sudden natural disasters occurred frequently, which not only caused heavy damages of casualties, property losses, and ecological environment but also brought serious harm to the society (Sheu, 2007b). To fight against the natural disasters, organizations like government need to transport relevant materials to the areas affected by the disasters. However, because of the factors like inadequate material preparation, untimely delivery, and blocked logistics, the relevant materials cannot be delivered to the right place on the right time, making the disaster harm further expand. In this situation, it is necessary to establish a complete disaster emergency logistics management system to prevent the further expansion of disasters (Sheu, 2007a).

Emergency logistics, a kind of special logistics providing emergency materials for various emergencies, aims to maximize the time efficiency and minimize disaster losses (Wang \& Xu, 2016). The effective management of emergency logistics can improve the ability of emergency logistics to deal with emergencies and the protection of people's life and property when natural disasters occur. The emergency logistics having good performance and high ability can ensure the efficiency of management (Wang \& Xu, 2016). The scientific and reasonable selection of emergency logistics suppliers is beneficial to establish long-term and stable partnership with good enterprises in emergency situations. Meanwhile, it also improves the timeliness of the whole material collection and reduces the uncertain risk in the process of emergency material procurement. Therefore, the selection of emergency logistics provider is important. To select the suppliers of emergency logistics, it is necessary to determine the evaluation indexes, which should follow the principles of systematization, science and conciseness (Wang \& Xu, 2016). Combined with the characteristics of emergency logistics, six criteria were selected, which are listed in Table 3. 
Table 3. Six criteria to select emergency logistics suppliers (Wang \& Xu, 2016)

\begin{tabular}{|l|l|}
\hline \multicolumn{1}{|c|}{ Criterion } & \multicolumn{1}{c|}{ Description } \\
\hline Product quality $\left(c_{1}\right)$ & $\begin{array}{l}\text { The quality of emergency supplies is the basic guarantee of emergency } \\
\text { rescue }\end{array}$ \\
\hline $\begin{array}{l}\text { Rapid response } \\
\text { capability }\left(c_{2}\right)\end{array}$ & $\begin{array}{l}\text { The ability to provide product information, order processing capability and } \\
\text { emergency delivery capability }\end{array}$ \\
\hline Supply capacity $\left(c_{3}\right)$ & The ability of suppliers to meet purchase orders in a timely manner \\
\hline Flexibility $\left(c_{4}\right)$ & $\begin{array}{l}\text { When the external demand changes, the supplier can respond to the } \\
\text { external demand at the first time and thus effectively meet the demand }\end{array}$ \\
\hline Price satisfaction $\left(c_{5}\right)$ & The purchase price advantage and stability of emergency supplies \\
\hline $\begin{array}{l}\text { Enterprise basic } \\
\text { management }\left(c_{6}\right)\end{array}$ & $\begin{array}{l}\text { The perfection of the enterprise's own emergency logistics plan system, } \\
\text { emergency drill, information level and its economic strength }\end{array}$ \\
\hline
\end{tabular}

Suppose that there are five alternative emergency logistics suppliers $a_{1}, a_{2}, a_{3}, a_{4}, a_{5}$. An expert $e$ evaluates the alternatives with PLTSs according to the six criteria $c_{1}, c_{2}, c_{3}, c_{4}, c_{5}, c_{6}$. The LTS is $S=\left\{s_{-3}=\right.$ Very Bad, $s_{-2}=$ Bad,$s_{-1}=A$ Little Bad, $s_{0}=$ Medium, $s_{1}=A$ Little Good, $s_{2}=$ Good, $s_{3}=$ Very Good $\}$. The probabilistic linguistic evaluation matrix $H=\left[h_{i j}(p)\right]_{5 \times 6}$ is shown as:

$\left[\begin{array}{cccccc}\left\{s_{1}(1)\right\} & \left\{s_{1}(0.4), s_{2}(0.6)\right\} & \left\{s_{2}(0.2), s_{3}(0.8)\right\} & \left\{s_{-1}(1)\right\} & \left\{s_{0}(0.2), s_{1}(0.8)\right\} & \left\{\begin{array}{l}s_{1}(0.1), s_{2}(0.4), \\ s_{3}(0.5)\end{array}\right\} \\ \left\{s_{2}(0.6), s_{3}(0.4)\right\} & \left\{\begin{array}{l}\left.s_{1}(0.3), s_{2}(0.4),\right\} \\ s_{3}(0.3)\end{array}\right\} & \left\{s_{1}(0.3), s_{2}(0.7)\right\} & \left\{s_{0}(0.2), s_{1}(0.8)\right\} & \left\{s_{-1}(0.7), s_{0}(0.3)\right\} & \left\{s_{3}(1)\right\}\end{array}\right\}$.

The pairwise comparison set of criteria and alternatives are $\aleph^{c}=\left\{<\left(c_{2}, c_{1}\right), 0.6>,<\left(c_{4}, c_{3}\right)\right.$, $\left.0.7>,<\left(c_{5}, c_{1}\right), 0.7>,<\left(c_{4}, c_{6}\right), 0.8>\right\}$ and $\aleph^{a}=\left\{<\left(a_{5}, a_{1}\right), 0.5>\right\}$.

\subsection{Implement the CUC method to solve the case}

According to Algorithm 1, Step 1 is given above, and Step 2 is performed as follows:

Step 2. The probabilistic linguistic evaluation matrix $H=\left[h_{i j}(p)\right]_{5 \times 6}$ is transformed to the probabilistic linguistic expected value matrix $\mathbf{E}=\left[E\left(h_{i j}(p)\right)\right]_{5 \times 6}$ by Eqs. (2)-(5) as follows:

$\left[\begin{array}{llllll}0.6860 & 0.7827 & 0.9694 & 0.2113 & 0.6488 & 0.9075 \\ 0.9083 & 0.8447 & 0.7988 & 0.6488 & 0.2979 & 1.0000 \\ 0.2451 & 0.2979 & 0.0734 & 0.9066 & 0.0918 & 0.1396 \\ 0.0184 & 0.6488 & 0.2113 & 0.1874 & 0.0092 & 0.0826 \\ 0.8302 & 1.0000 & 0.6488 & 0.7344 & 0.9694 & 0.6302\end{array}\right]$.

Step 3. Let the criterion preference ratios be $\kappa_{2,1}, \kappa_{3,1}, \kappa_{4,1}, \kappa_{5,1}$ and $\kappa_{6,1}$. Set up Model 1 . 


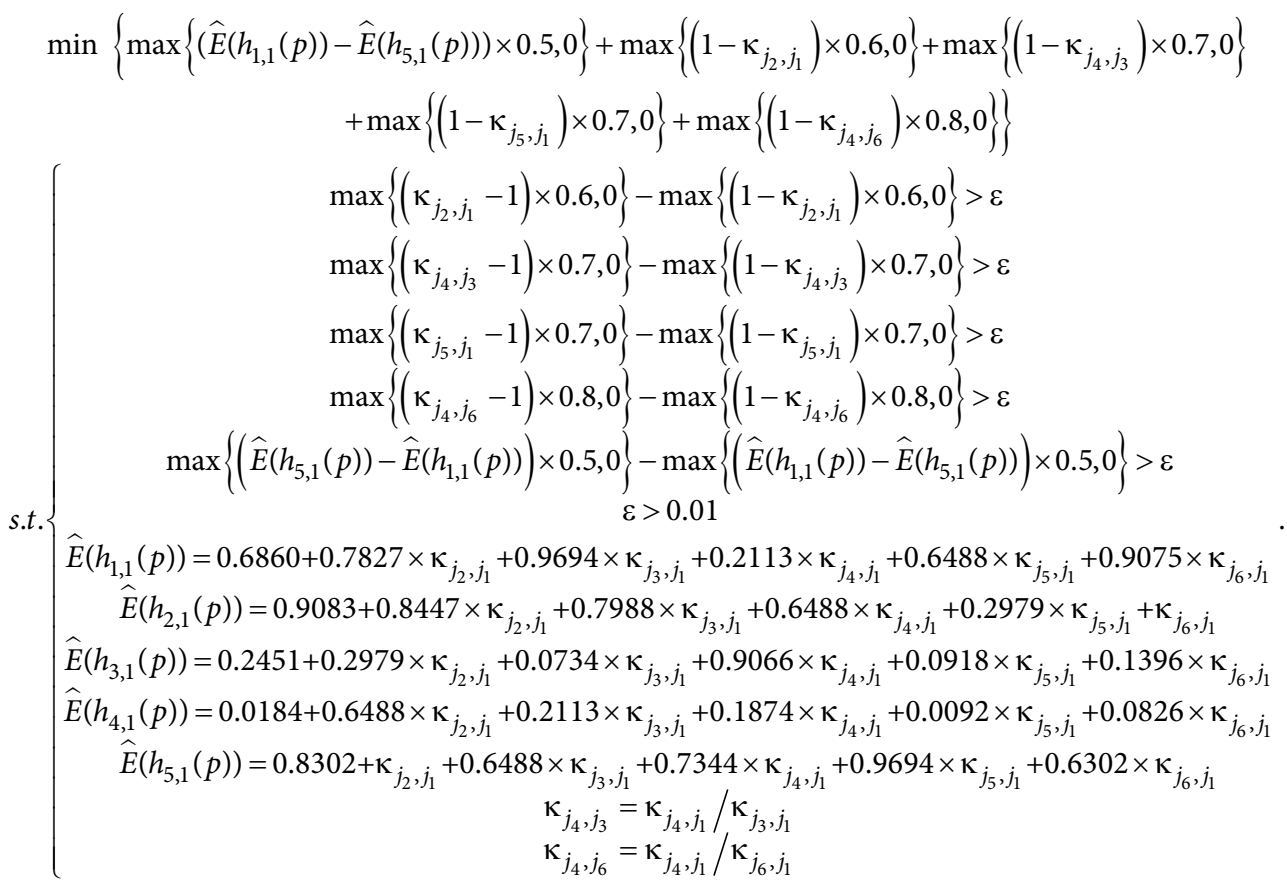

Step 4. Solving Model 1, the criterion preference ratios are $\kappa_{2,1}=5.0336, \kappa_{3,1}=0.6029$, $\widehat{E}\left(h_{41}(p)\right)=4.478, \kappa_{5,1}=4.4797, \kappa_{6,1}=1.2224 . \kappa_{2,1}>1$ means that the expert prefers $c_{j_{2}}$ to $c_{j_{1}}$, which is consistent with the preconditions. The adjusted probabilistic linguistic expected values can be calculated as $\widehat{E}\left(h_{11}(p)\right)=10.269, \hat{E}\left(h_{21}(p)\right)=11.399, \widehat{E}\left(h_{31}(p)\right)=6.843$, $\widehat{E}\left(h_{41}(p)\right)=4.478$ and $\widehat{E}\left(h_{51}(p)\right)=14.991$. Finally, the alternatives are ranked according to the probabilistic linguistic expected values as $a_{5} \succ a_{2} \succ a_{1} \succ a_{3} \succ a_{4}$. The fifth emergency logistics supplier $a_{5}$ should be selected because it is the best among the five alternatives.

\subsection{Comparative analyses and discussions}

The PL-LINMAP method (Liao et al., 2019a) is a linear programming model based on incomplete preference information to solve MCDM problems in the probabilistic linguistic environment. We use the PL-LINMAP method to solve this case and compare the results of the PL-LINMAP method.

The PL-LINMAP method just uses the alternative preference information $\aleph^{a}=\left\{<\left(a_{5}, a_{1}\right), 0.5>\right\}$. The results of the PL-LINMAP method are $w=(0,0.88,0,0,0,0.12)$ and $A^{+}=\left\{\left\{s_{-3}(1)\right\},\left\{s_{-2}(1)\right\},\left\{s_{-3}(1)\right\},\left\{s_{-3}(1)\right\},\left\{s_{-3}(1)\right\},\left\{s_{-2}(1)\right\}\right\} . w$ are the weights of the six criteria. $A^{+}$are the evaluations of the positive ideal supplier under the six criteria. The final ranking of the alternatives is $a_{2} \succ a_{3} \succ a_{1} \succ a_{4} \succ a_{5} . a_{2}$ is the best emergency logistics supplier among the five alternatives.

In addition to the PL-LINMAP method, the PL-MULTIMOOR method (Wu et al., 2018), PL-CoCoSo method (Wen et al., 2019), and PL-TOPSIS method (Lu et al., 2020) are also employed to solve the case, and the results are shown in Table 4. 
Table 4 . The results derived by different PL-MCDM methods

\begin{tabular}{|l|c|c|c|c|c|c|}
\hline \multicolumn{1}{|c|}{ Methods } & $a_{1}$ & $a_{2}$ & $a_{3}$ & $a_{4}$ & $a_{5}$ & Rankings \\
\hline PL-MULTIMOOR & 0.054 & 0.097 & -0.001 & -0.015 & 0.179 & $a_{5} \succ a_{2} \succ a_{1} \succ a_{3} \succ a_{4}$ \\
\hline PL-CoCoSo & 2.423 & 2.865 & 1.426 & 0.698 & 3.278 & $a_{5} \succ a_{2} \succ a_{1} \succ a_{3} \succ a_{4}$ \\
\hline PL-TOPSIS & 0.502 & 0.382 & 0.789 & 0.914 & 0.452 & $a_{2} \succ a_{5} \succ a_{1} \succ a_{3} \succ a_{4}$ \\
\hline PL-LINMAP & 6.332 & 4.329 & 4.726 & 9.225 & 29.867 & $a_{2} \succ a_{3} \succ a_{1} \succ a_{4} \succ a_{5}$ \\
\hline PL-CUC & 10.269 & 11.339 & 6.843 & 4.478 & 14.991 & $a_{5} \succ a_{2} \succ a_{1} \succ a_{3} \succ a_{4}$ \\
\hline
\end{tabular}

The calculation results of the PL-LINMAP method are quite different from those of the PL-CUC method. The results of the PL-TOPSIS method are slightly different from those of the PL-CUC method. The calculation results of the PL-MULTIMOOR method and PLCoCoSo method are consistent with the PL-CUC method. The reasons for the differences in results and the advantages of the PL-CUC method are discussed as follows:

(1) Low cognitive burden. The PL-CUC method only requires experts to give a part of preference information of criteria or alternatives, and the ranking of alternatives can be deduced from such preference information. The PL-MULTIMOOR method and PL-CoCoSo method require complete preference information of criteria to get the weights of criteria and the ranking of alternatives. Because the PL-CUC method needs less preference information than the PL-MULTIMOOR method and PL-CoCoSo method to get the ranking of alternatives, the proposed method can reduce the cognitive burdens of experts in decision-making.

(2) High reliability. The differences in the results of the PL-TOPSIS method, PL-MULTIMOOR method, and PL-CoCoSo method result from the differences in their aggregation methods. The PL-MULTIMOOR method and PL-CoCoSo method use three aggregation strategies while the PL-TOPSIS method has only one aggregation method, so the robustness of the PL-MULTIMOOR and PL-CoCoSo methods is stronger than that of the PL-TOPSIS method. As the results of the PL-MULTIMOOR, PL-CoCoSo, and PL-CUC methods are the same rankings, the reliability of the PL-CUC method is higher than that of the PL-TOPSIS method. In addition, the weights of the first, third, fourth, and fifth criteria calculated by the PL-LINMAP method are 0 , leading to that the result of the PL-LINMAP method is different from that of the PL-CUC method. Because the weights having the value 0 make part of the evaluations unused, the result of the PL-LINMAP method is less reliable than that of the PL-CUC method. In a nutshell, the PL-CUC method has higher reliability than the PL-TOPSIS method and PL-LINMAP method.

(3) Strong applicability. The PL-LINMAP method, PL-MULTIMOOR method, PL-CoCoSo method, and PL-TOPSIS method cannot be used to deal with the MCDM problem where the criteria are relevant. For the PL-CUC method, the relevance of criteria is used to get the criterion preference ratios. In view of this, the PL-CUC method has stronger applicability than other methods in Table 4. 


\section{Conclusions}

This study proposed a criteria utility conversion (CUC) technique to solve probabilistic linguistic MCDM problems. To sum up, the contributions of this paper are as follows: 1) The criterion preference ratio was defined. By considering the trade-offs of the utilities of evaluations among criteria, the utility conversion process was defined from the even swaps method. Based on the utility conversion process, the criterion preference ratio was defined to express the preference relations of experts to criteria. 2) The PL-CUC method was proposed. A linear programming problem aiming to minimize the total criterion inconsistency and total alternative inconsistency was set up to calculate the criterion preference ratios. The criterion preference ratios and the linguistic scale functions of PLTSs were applied to get the ranking of alternatives. 3) The feasibility of the PL-CUC method with probabilistic linguistic information was verified by solving the case of selecting emergency logistics supplier. The comparative analysis shows that the PL-CUC method has the advantages of low cognitive burden, high stability, and strong applicability.

In future study, we will consider to extend the criterion utility conversion process and obtain other forms of criterion utility conversion process under the assumption that experts are not completely rational. Meanwhile, the CUC method can also be applied to other practical fields.

\section{Funding}

The work was supported by the National Natural Science Foundation of China under Grant [numbers 71771156, 71971145].

\section{Author contributions}

Rui QIN and Huchang LIAO conceived the study and were responsible for the design and development of the data analysis. Rui QIN and Lisheng JIANG were responsible for data collection and analysis. Rui QIN and Huchang LIAO were responsible for data interpretation. Rui QIN and Lisheng JIANG wrote the first draft of the article. Huchang LIAO checked and revise the draft.

\section{Disclosure statement}

The authors have no competing financial, professional, or personal interests from other parties that are related to this paper.

\section{References}

Adhami, A. Y., \& Ahmad, F. (2020). Interactive Pythagorean-hesitant fuzzy computational algorithm for multiobjective transportation problem under uncertainty. International Journal of Management Science and Engineering Management, 15(4), 288-297.

https://doi.org/10.1080/17509653.2020.1783381 
Altun, K., \& Dereli, T. (2014). Even easier multi-issue negotiation through modified Even-Swaps considering practically dominated alternatives. Computers \& Industrial Engineering, 76, 307-317. https://doi.org/10.1016/j.cie.2014.08.015

Ben Abdelaziz, F., Lang, P., \& Nadeau, R. (1999). Dominance and efficiency in multicriteria decision under uncertainty. Theory and Decision, 47(3), 191-211. https://doi.org/10.1023/A:1005102326115

Chen, S. X., Wang, J. Q., \& Wang, T. L. (2019). Cloud-based ERP system selection based on extended probabilistic linguistic MULTIMOORA method and Choquet integral operator. Computational \& Applied Mathematics, 38(2), 88. https://doi.org/10.1007/s40314-019-0839-Z

Chen, T. Y. (2019). Multiple criteria group decision making using a parametric linear programming technique for multidimensional analysis of preference under uncertainty of Pythagorean fuzziness. IEEE Access, 7, 174108-174128. https://doi.org/10.1109/ACCESS.2019.2957161

Dereli, T., \& Altun, K. (2012). Modified Even-Swaps: A novel, clear, rational and an easy-to-use mechanism for multi-issue negotiation. Computers \& Industrial Engineering, 63(4), 1013-1029. https://doi.org/10.1016/j.cie.2012.06.013

Elahi, G., \& Yu, E. (2012). Comparing alternatives for analyzing requirements trade-offs - In the absence of numerical data. Information and Software Technology, 54(6), 517-530. https://doi.org/10.1016/j.infsof.2011.10.007

Fang, R., Liao, H. C., Yang, J. B., \& Xu, D. L. (2021). Generalised probabilistic linguistic evidential reasoning approach for multi-criteria decision-making under uncertainty. Journal of the Operations Research Society, 72(1), 130-144. https://doi.org/10.1080/01605682.2019.1654415

Gomes, L. F. A. M., Rangel, L. A. D., \& Fernandes, P. P. (2012). Decision aiding in plastic surgery: a multicriteria analysis. Pesquisa Operacional, 32(2), 371-387.

https://doi.org/10.1590/S0101-74382012005000011

Haghighi, M. H., Mousavi, S. M., \& Mohagheghi, V. (2019). A new soft computing model based on linear assignment and linear programming technique for multidimensional analysis of preference with interval type-2 fuzzy sets. Applied Soft Computing, 77, 780-796.

https://doi.org/10.1016/j.asoc.2019.01.048

Hamidzadeh, Z., Sattari, S., Soltanieh, M., \& Vatani, A. (2020). Development of a multi-objective decision-making model to recover flare gases in a multi flare gases zone. Energy, 203, 117815. https://doi.org/10.1016/j.energy.2020.117815

Hammond, J. S., Keeney, R. L., \& Raiffa, H. (1993). Even Swaps: a rational method for making tradeoffs. Harvard Business Review, 76(2), 137-138. https://www.researchgate.net/publication/13119982

Haseli, G., Sheikh, R., \& Sana, S. S. (2020). Base-criterion on multi-criteria decision-making method and its applications. International Journal of Management Science and Engineering Management, 15(2), 79-88. https://doi.org/10.1080/17509653.2019.1633964

Kajanus, M., Ahola, J., Kurttila, M., \& Pesonen, M. (2001). Application of even swaps for strategy selection in a rural enterprise. Management Decision, 39(5), 394-402.

https://doi.org/10.1108/00251740110395688

Kashef, M., Safari, H., Maleki, M., \& Cruz-Machado, V. (2018). Solving MCDM problems based on combination of PACMAN and LINMAP. Journal of Multi-Criteria Decision Analysis, 25(5-6), 169-176. https://doi.org/10.1002/mcda.1650

Lahtinen, T. J., \& Hämäläinen, R. P. (2016). Path dependence and biases in the even swaps decision analysis method. European Operations Research Perspectives, 249(3), 890-898. https://doi.org/10.1016/j.ejor.2015.09.056

Lahtinen, T. J., Hämäläinen, R. P., \& Jenytin, C. (2020). On preference elicitation processes which mitigate the accumulation of biases in multi-criteria decision analysis. European Journal of Operational Research, 282(1), 201-210. https://doi.org/10.1016/j.ejor.2019.09.004

Lei, F., Wei, G. W., Wu, J., Wei, C., \& Guo, Y. F. (2020). QUALIFLEX method for MAGDM with probabilistic uncertain linguistic information and its application to green supplier selection. Journal of Intelligent \& Fuzzy Systems, 39(5), 6819-6831. https://doi.org/10.3233/JIFS-191737 
Li, D. F. (2008). Extension of the LINMAP for multiattribute decision making under Atanassov's intuitionistic fuzzy environment. Fuzzy Optimization and Decision Making, 7(1), 17-34. https://doi.org/10.1007/s10700-007-9022-x

Li, D. F., \& Wan, S. P. (2014). Fuzzy heterogeneous multiattribute decision making method for outsourcing provider selection. Expert Systems with Applications, 41(6), 3047-3059. https://doi.org/10.1016/j.eswa.2013.10.036

Li, H. L., \& Ma, L. C. (2008). Visualizing decision process on spheres based on the even swap concept. Decision Support Systems, 45(2), 354-367. https://doi.org/10.1016/j.dss.2008.01.004

Liao, H. C., Jiang, L. S., Lev, B., \& Fujita, H. (2019a). Novel operations of PLTSs based on the disparity degrees of linguistic terms and their use in designing the probabilistic linguistic ELECTRE III method. Applied Soft Computing, 80, 450-464. https://doi.org/10.1016/j.asoc.2019.04.018

Liao, H. C., Jiang, L. S., Xu, Z. S., Xu, J. P., \& Herrera, F. (2017). A linear programming method for multiple criteria decision making with probabilistic linguistic information. Information Science, 415, 341-355. https://doi.org/10.1016/j.ins.2017.06.035

Liao, H. C., Mi, X. M., \& Xu, Z. S. (2020). A survey of decision-making methods with probabilistic linguistic information: Bibliometrics, preliminaries, methodologies, applications and future directions. Fuzzy Optimization and Decision Making, 19(1), 81-134.

https://doi.org/10.1007/s10700-019-09309-5

Liao, Z. Q., Liao, H. C., Gou, X. J., Xu, Z. S., \& Zavadskas, E. K. (2019b). A hesitant fuzzy linguistic choquet integral-based MULTIMOORA method for multiple criteria decision making and its application in talent selection. Economic Computation and Economic Cybernetics Studies and Research, 53(2), 113-130. https://doi.org/10.24818/18423264/53.2.19.07

Lin, M. W., Chen, Z. Y., Liao, H. C., \& Xu, Z. S. (2019). ELECTRE II method to deal with probabilistic linguistic term sets and its application to edge computing. Nonlinear Dynamics, 96(3), 2125-2143. https://doi.org/10.1007/s11071-019-04910-0

Lu, X. Y., Dong, J. Y., \& Wan, S. P. (2020). A novel three-phase LINMAP method for hybrid multi-criteria group decision making with dual hesitant fuzzy truth degrees. IEEE Access, 8, 112462-112483. https://doi.org/10.1109/ACCESS.2020.3001941

Mehrabadi, Z. K., \& Boyaghchi, F. A. (2019). Thermodynamic, economic and environmental impact studies on various distillation units integrated with gasification-based multi-generation system: comparative study and optimization. Journal of Cleaner Production, 241, 118333. https://doi.org/10.1016/j.jclepro.2019.118333

Mi, X. M., Liao, H. C., Wu, X. L., \& Xu, Z. S. (2020). Probabilistic linguistic information fusion: a survey on aggregation operators in terms of principles, definitions, classifications, applications and challenges. International Journal of Intelligent Systems, 35(3), 529-556. https://doi.org/10.1002/int.22216

Milutinovic, G., Ahonen-Jonnarth, U., \& Seipel, S. (2018). GISwaps: a new method for decision making in continuous choice models based on even swaps. International Journal of Decision Support Systems Technology, 10(3), 57-78. https://doi.org/10.4018/IJDSST.2018070104

Mustajoki, J., \& Hämäläinen, R. P. (2005). A preference programming approach to make the even swaps method even easier. Decision Analysis, 2(2), 110-123. https://doi.org/10.1287/deca.1050.0043

Mustajoki, J., \& Hämäläinen, R. P. (2007). Smart-Swaps - A decision support system for multicriteria decision analysis with the even swaps method. Decision Support Systems, 44(1), 313-325. https://doi.org/10.1016/j.dss.2007.04.004

Pang, Q., Wang, H., \& Xu, Z. S. (2016). Probabilistic linguistic term sets in multi-attribute group decision making. Information Sciences, 369, 128-143. https://doi.org/10.1016/j.ins.2016.06.021

Rodríguez, R. M., Martıńez, L., \& Herrera, F. (2012). Hesitant fuzzy linguistic terms sets for decision making. IEEE Transactions on Fuzzy Systems, 20(1), 109-119.

https://doi.org/10.1109/TFUZZ.2011.2170076 
Shaikh, A., Singh, A., Ghose, D., \& Shabbiruddin. (2020). Analysis and selection of optimum material to improvise braking system in automobiles using integrated Fuzzy-COPRAS methodology. International Journal of Management Science and Engineering Management, 15(4), 265-273. https://doi.org/10.1080/17509653.2020.1772895

Sheu, J. B. (2007a). Challenges of emergency logistics management. Transportation Research Part ELogistics and Transportation Review, 43(6), 655-659. https://doi.org/10.1016/j.tre.2007.01.001

Sheu, J. B. (2007b). An emergency logistics distribution approach for quick response to urgent relief demand in disasters. Transportation Research Part E-Logistics and Transportation Review, 43(6), 687-709. https://doi.org/10.1016/j.tre.2006.04.004

Srinivasan, V., \& Shocker, A. D. (1973). Linear programming techniques for multidimensional analysis of preference. Psychometrica, 38, 337-342. https://doi.org/10.1007/BF02291658

Wang, J.-C., \& Chen, T.-Y. (2020). A novel Pythagorean fuzzy LINMAP-based compromising approach for multiple criteria group decision-making with preference over alternatives. International Journal of Computational Intelligence Systems, 13(1), 444-463. https://doi.org/10.2991/ijcis.d.200408.001

Wang, J.-Q., Wu, J.-T., Wang, J. H., Zhang, H.-Y., \& Chen, X.-H. (2014). Interval-valued hesitant fuzzy linguistic sets and their applications in multi-criteria decision-making problems. Information Sciences, 288, 55-72. https://doi.org/10.1016/j.ins.2014.07.034

Wang, L., \& Xu, J. Z. (2016). Emergency logistics distribution optimization model and algorithm in disaster chain. Logistics Technology, 35(8), 115-118.

Wei, G. W., Lei, F., Lin, R., Wang, R., Wei, Y., Wu, J., \& Wei, C. (2020). Algorithms for probabilistic uncertain linguistic multiple attribute group decision making based on the GRA and CRITIC method: application to location planning of electric vehicle charging stations. Economic Research-Ekonomska Istraživanja, 33(1), 828-846. https://doi.org/10.1080/1331677X.2020.1734851

Wen, Z., Liao, H. C., Ren, R. X., Bai, C. G., Zavadskas, E. K., Antucheviciene, J., \& Al-Barakati, A. (2019). Cold chain logistics management of medicine with an integrated multi-criteria decisionmaking method. International Journal of Environmental Research and Public Health, 16(23), 4843. https://doi.org/10.3390/ijerph16234843

Wu, X. L., \& Liao, H. C. (2019). A consensus-based probabilistic linguistic gained and lost dominance score method. European Journal of Operational Research, 272(3), 1017-1027. https://doi.org/10.1016/j.ejor.2018.07.044

Wu, X. L., Liao, H. C., Xu, Z. S., Hafezalkotob, A., \& Herrera, F. (2018). Probabilistic linguistic MULTIMOORA: a multicriteria decision making method based on the probabilistic linguistic expectation function and the improved borda rule. IEEE Transactions on Fuzzy Systems, 26(6), 3688-2702. https://doi.org/10.1109/TFUZZ.2018.2843330

Yao, Q. Z. (2019). Multi-objective optimization design of spur gear based on NSGA-II and decision making. Advances in Mechanical Engineering, 11(3), 1-8. https://doi.org/10.1177/1687814018824936

Zadeh, L. A. (1975). The concept of a linguistic variable and its applications to approximate reasoningPart I. Information Sciences, 8, 199-249. https://doi.org/10.1016/0020-0255(75)90036-5

Zhang, X. L., \& Xing, X. M. (2017). Probabilistic linguistic VIKOR method to evaluate green supply chain initiatives. Sustainability, 9(7), 1231. https://doi.org/10.3390/su9071231

Zuo, W. J., Li, D. F., Yu, G. F., \& Zhang, L. P. (2019). A large group decision-making method and its application to the evaluation of property perceived service quality. Journal of Intelligent \& Fuzzy Systems, 37(1), 1513-1527. https://doi.org/10.3233/JIFS-182934 